\title{
Critical Magnetic Scattering of Neutrons in Iron
}

\author{
Passell, L.; Blinowski, K.; Brun, T.; Nielsen, P.
}

\section{Published in:}

PHYSICAL REVIEW

Link to article, DOI:

10.1103/PhysRev.139.A1866

Publication date:

1965

Document Version

Publisher's PDF, also known as Version of record

Link back to DTU Orbit

\section{Citation (APA):}

Passell, L., Blinowski, K., Brun, T., \& Nielsen, P. (1965). Critical Magnetic Scattering of Neutrons in Iron. PHYSICAL REVIEW, 139(6A), A1866-A1876. https://doi.org/10.1103/PhysRev.139.A1866

\section{General rights}

Copyright and moral rights for the publications made accessible in the public portal are retained by the authors and/or other copyright owners and it is a condition of accessing publications that users recognise and abide by the legal requirements associated with these rights.

- Users may download and print one copy of any publication from the public portal for the purpose of private study or research.

- You may not further distribute the material or use it for any profit-making activity or commercial gain

- You may freely distribute the URL identifying the publication in the public portal

If you believe that this document breaches copyright please contact us providing details, and we will remove access to the work immediately and investigate your claim 


\title{
Critical Magnetic Scattering of Neutrons in Iron
}

\author{
L. PASSEll* and K. Blinowski $\dagger$ \\ Research Establishment Ris $\phi$, Roskilde, Denmark \\ AND \\ T. BRUN $\ddagger$ \\ Technical University, Copenhagen, Denmark \\ AND \\ P. NiELSEN \\ University of Copenhagen, Physical Laboratory II, Copenhagen, Denmark
}

(Received 29 April 1965)

\begin{abstract}
Measurements of the angular and energy distributions of $4.28 \AA$ neutrons scattered at small angles from iron at temperatures above the Curie temperature are described. The results are interpreted in terms of Van Hove's theory of critical magnetic scattering and yield information on the range of spin correlations and the dynamics of the spin-ordering process. For the dimensionless parameter $2 m \Delta / \hbar$ which describes the time dependence of the spin fluctuations, we obtain the value $11.0 \pm 0.6$ at $T-T_{c}=2$ and $18^{\circ} \mathrm{C}$. The zero-field magnetic susceptibility, as determined by the parameters $\kappa_{1}$ and $r_{1}$ (which represent the range and strength, respectively, of the spin correlations), is found to vary as $\left(T-T_{c}\right)^{-1.20 \pm 0.04}$. Near the Curie temperature there was sufficient intensity to measure the ratio of the coefficients of the $K^{4}$ and $K^{2}$ terms of the angular distribution. The value of this ratio, $29 \AA^{2}$, is related to the existence of long-range couplings within the spin system. Details of certain recent modifications of the theory of critical systems are discussed and compared with the experimental results.
\end{abstract}

\section{INTRODUCTION}

$\mathbf{A}^{\mathrm{L}}$ LTHOUGH slow neutrons are now very commonly used for studies of the structure and dynamics of magnetic systems, their application to the study of magnetic phase transitions is fairly recent. Historically, it had its beginnings when Cassels and Latham ${ }^{1}$ investigated the cross section of iron at high temperatures. They noted that the cross section was appreciably larger than could be accounted for by temperature diffuse scattering alone, and while the evidence was then far from conclusive, they suggested that the difference was in some way associated with the magnetic properties of iron. Later investigations with long-wavelength neutrons $^{2,3}$ showed that the cross section actually had a pronounced peak at the Curie temperature, thus establishing that the interaction was clearly of magnetic origin. The specific mechanism responsible was not understood, however, until Van Hove $e^{4}$ pointed out that the largescale fluctuations in the density of magnetization, which occur in the neighborhood of the ordering temperature, will scatter neutrons in much the same way that light is scattered by liquids and dense gases under critical conditions.

Van Hove subsequently developed a theory of mag-

* Present address: Brookhaven National Laboratory, Upton, New York.

$\dagger$ Present address: Institute of Nuclear Research, Swierk k. Otwocka, Poland.

$\ddagger$ Present address: Institute for Atomic Research, Iowa State University, Ames, Iowa.

1 J. M. Cassels and R. Latham, Phys. Rev. 74, 103 (1948); Proc. Phys. Soc. A65, 241 (1952).

${ }^{2}$ D. J. Hughes and H. Palevsky, Phys. Rev. 92, 202 (1954).

3 G. L. Squires, Proc. Phys. Soc. A67, 248 (1954).

${ }^{4}$ L. Van Hove, Phys. Rev. 93, 268 (1954). netic critical scattering ${ }^{5}$ based on the Ornstein-Zernike ${ }^{6}$ treatment (as extended by Klein and Tisza) of the critical scattering of light in gases. He showed that there was a direct relationship between spin correlations in the scatterer and the angular and energy distribution of the scattered neutrons and predicted that there would be intense scattering around Bragg peaks at the ordering temperature. This scattering was soon observed in iron by Wilkinson, Shull, and Gersch ${ }^{7}$ and by Lowde ${ }^{8}$ and in antiferromagnetic cobalt oxide by McReynolds and Riste. ${ }^{9}$ These measurements, and others which followed ${ }^{10-13}$ demonstrated that neutron scattering could give considerable information on the magnetic ordering process.

In his original formulation of the theory, Van Hove pointed out that the parameters which describe the scattering of neutrons can be related to the magnetic susceptibility of the scattering system by general statistical-mechanical considerations. Neutron-scattering measurements thus serve to determine the zero-field magnetic susceptibility of the scatterer in the neighbor-

\footnotetext{
${ }^{6}$ L. Van Hove, Phys. Rev. 95, 249 (1954); ibid. 95, 1374 (1954).

' L. S. Ornstein and F. Zernike, Proc. Acad. Sci. Amsterdam 17, 793 (1914).

${ }^{7}$ H. A. Gersch, C. G. Shull, and M. K. Wilkinson, Phys. Rev. 103,525 (1956).

R. D. Lowde, Rev. Mod. Phys. 30, 69 (1958).

$\checkmark$ A. W. McReynolds and T. Riste, Bull. Am. Phys. Soc. 1, 133 (1956); Phys. Rev. 95, 116 (1957).

${ }^{10} \mathrm{~T}$. Riste, K. Blinowski, and J. Janik, J. Phys. Chem. Solids 9, 153 (1959).

11 M. Ericson and B. Jacrot, J. Phys. Chem. Solids 13, 235 (1960).

${ }^{12}$ T. Riste, J. Phys. Chem. Solids 17, 308 (1961)

13 B. Jacrot, J. Konstantinovic, G. Parette, and D. Cribier Symposium on Inelastic Scattering of Neutrons in Solids and Liquids, Chalk River, 1962 (unpublished).
} 
hood of the ordering temperature. Recently, Domb and Sykes $^{14}$ and Gammel, Marshall, and Morgan ${ }^{15}$ have developed theories of the temperature dependence of the susceptibility of a Heisenberg ferromagnet at temperatures slightly above the Curie temperature. Both theories predict that the susceptibility should vary as $\left(T-T_{c}\right)^{-4 / 3}$ rather than $\left(T-T_{c}\right)^{-1}$ as predicted by the older molecular-field and Bethe-Peierls-Weiss models. Although there is some question as to whether the Heisenberg model completely applies to metals, the low-field susceptibility measurements on iron made by Noakes and Arrott ${ }^{16}$ and Arajs and Colvin ${ }^{17}$ and the critical-scattering measurements of Jacrot, Konstantinovic, Parette, and $\mathrm{Cribier}^{13}$ both appear to fit the $\frac{4}{3}$ law.

This paper describes an investigation of critical magnetic neutron scattering in iron which we have recently completed. Since we were particularly interested in comparing the theoretical predictions mentioned above with experiment, we made most of our measurements in the temperature region $0<T-T_{c}<60^{\circ} \mathrm{C}$ covering both the temperature range over which the new theories are expected to hold and the higher temperature transition region in which the temperature dependence of the susceptibility is expected to approach the linear behavior predicted by the earlier models.

\section{THE CRITICAL-SCATTERING CROSS SECTION}

Excellent reviews of the theory of critical neutron scattering are available in the articles by de Gennes, ${ }^{18}$ by Izyumov, ${ }^{19}$ and by Van Hove. ${ }^{5}$ In Van Hove's formulation the magnetic scattering cross section for a polycrystal of $N$ spins is expressed as the Fourier transform over $r$ and $t$ of the pair distribution in space and time I $(\mathbf{r}, t)$, i.e.,

$$
\begin{aligned}
\frac{d^{2} \sigma}{d \Omega d E}=\left(\frac{\gamma e^{2}}{m_{e} c^{2}}\right)^{2} & \frac{N}{3 \pi \hbar} \frac{k}{k_{0}} F|(\mathbf{K})|^{2} \\
& \times \int \exp [i(\mathbf{K} \cdot \mathbf{r}-\omega t)] \Gamma(\mathbf{r}, t) d \mathbf{r} d t .
\end{aligned}
$$

Here $\mathbf{k}_{0}$ and $\mathbf{k}=\mathbf{k}_{0}-\mathbf{K}$ are the initial and final wave vectors of the neturon, $E$ is the scattered neutron energy, $\omega=\hbar\left(k_{0}^{2}-k^{2}\right) / 2 m, \gamma$ and $m$ are the neutron magnetic moment and mass, respectively, and $F(\mathbf{K})$ is the form factor for magnetic electrons.

${ }^{14}$ C. Domb and M. F. Sykes, Phys. Rev. 128, 168 (1962).

${ }^{15} \mathrm{~J}$. L. Gammel, W. Marshall, and L. Morgan, Proc. Roy. Soc. (London) A275, 257 (1963).

${ }_{16} \mathrm{~J}$. E. Noakes and A. Arrott, J. Appl. Phys. Suppl. 35, 931 (1964).

${ }_{17}$ S. Arajs and R. V. Colvin, J. Appl. Phys. Suppl. 35, 2424 (1964)

${ }_{18}$ P. G. de Gennes, Magnetism, edited by G. T. Rado and H. Suhl (Academic Press Inc., New York, 1963), Vol. III, p. 115

${ }_{19} \mathrm{Yu}$. A. Izyumov, Uspekhi Fiz. Nauk 80, 41 (1963) [English transl.: Soviet Phys.-Uspekhi 16, 359 (1963)].
All of the essential physical description of the scatterer is contained in the pair distribution function which is written in the form

$$
\Gamma(\mathbf{r}, t)=\sum_{R} \gamma_{R}(t) G_{R}(\mathbf{r}, t) .
$$

$G_{R}(\mathbf{r}, t)$ represents the pair distribution of two atoms with equilibrium positions separated by $\mathbf{R}$ and $\gamma_{R}(t)$ $=\left\langle\mathbf{S}_{0}(0) \cdot \mathbf{S}_{R}(t)\right\rangle_{T}$, represents the correlation between the corresponding pairs of spins. Customarily $\gamma_{R}(t)$ is expressed as the sum of two terms

$$
\gamma_{R}(t)=\left|\left\langle S_{T}\right\rangle\right|^{2}+\gamma_{R}{ }^{\prime}(t) .
$$

The first term, which is the square of the average spin vector per atom, gives rise to the elastic magnetic Bragg scattering, while the second term, which represents the time-dependent deviation of $\gamma_{R}(t)$ from its asymptotic value, gives an additional inelastic diffuse magnetic scattering. It is this inelastic diffuse scattering which becomes the intense critical scattering observed in the neighborhood of the ordering temperature.

If atomic displacements are neglected, $G_{R}(\mathbf{r}, t)$ becomes simply $\delta(\mathbf{r}-\mathbf{R})$, in which case the integration over $\mathbf{r}$ in (1) is replaced by a summation over lattice sites $\mathbf{R}$. Thus, the critical scattering cross section can be written as

$$
\begin{aligned}
\frac{d^{2} \sigma}{d \Omega d E}=\left(\frac{\gamma e^{2}}{m_{e} c^{2}}\right)^{2} & \frac{N}{3 \pi h} \frac{k}{k_{0}}|F(\mathbf{K})|^{2} \\
& \times \sum_{R} \int \exp [i(\mathbf{K} \cdot \mathbf{R}-\omega t)] \gamma_{R}(t) d t .
\end{aligned}
$$

If for the moment we ignore the time dependence of this expression, the $\sum_{R}$ in (4) is by definition $\chi(\mathbf{K})$, the static susceptibility of the spin system for a sinusoidal perturbation of wave vector $\mathbf{K}$. Thus,

$$
\chi(\mathbf{K}) / \chi_{1} \equiv(S(S+1))^{-1} \sum_{R} \gamma_{R}(0) \exp (i \mathbf{K} \cdot \mathbf{R}),
$$

where $\chi_{1}$ represents $N g^{2} \mu^{2} S(S+1) / 3 k T$, which is the susceptibility of a system of noninteracting ions. Above the Curie temperature all that remains of the correlation function $\gamma_{R}(t)$ is the fluctuation term which, in its time-independent form, can be identified with the instantaneous correlation function of the OrnsteinZernike theory ${ }^{6}$

$$
\gamma_{R^{\prime}}(0)=\frac{V_{0} S(S+1)}{4 \pi r_{1}{ }^{2}} \frac{\exp \left(-\kappa_{1} R\right)}{R} .
$$

Here $V_{0}$ is the volume of the crystal cell and the parameters $\kappa_{1}^{-1}$ and $r_{1}$ represent, respectively, the range and strength of the spin correlations. It is important to note that (6) is an asymptotic expression valid in the limit of $\kappa_{1}^{-1}$ and $R$ large compared to interatomic distances. The relationship between the scattering parameters and the susceptibility can be found by substituting (6) into 
(5) which leads to the formula

$$
\left(\kappa_{1} r_{1}\right)^{2}=\chi_{1} / \chi(0) \text {. }
$$

To determine the time dependence of $\gamma_{R}{ }^{\prime}(t)$, Van Hove assumed that the equation for the density of magnetization is of the diffusion type. It then follows that the decay in time of a plane-wave fluctuation of the magnetization is given by $\exp \left(-\Lambda q^{2} t\right)$ with $q$ the wave vector and where $\boldsymbol{\Lambda}$ is the diffusion constant (which is inversely proportional to the magnetic susceptibility). Thus each Fourier component of $\gamma_{R}{ }^{\prime}(t)$ will decay as $\exp \left(-\Lambda q^{2} t\right)$ and $\gamma_{R}{ }^{\prime}(t)$ can be expressed as a Fourier integral

$$
\gamma_{R^{\prime}}(t)=\int_{-\infty}^{\infty} \alpha(\mathbf{q}) \exp (i \mathbf{q} \cdot \mathbf{R}) \exp \left(-\Lambda q^{2} t\right) d \mathbf{q},
$$

where $\alpha(\mathbf{q})$ is determined by the condition that $\gamma_{R}{ }^{\prime}(0)$ is the Ornstein-Zernike function (6). With the help of the Fourier convolution theorem, (8) becomes

$$
\begin{aligned}
& \gamma_{R^{\prime}}(t)=\frac{V_{0} S(S+1)}{4 \pi r_{1}{ }^{2}(4 \pi \Lambda|t|)^{3 / 2}} \\
& \quad \times \int \exp \left(-\frac{\left|\mathbf{R}-\mathbf{R}^{\prime}\right|}{4 \Lambda t}-\kappa_{1} R^{\prime}\right) \frac{d \mathbf{R}^{\prime}}{R^{\prime}} .
\end{aligned}
$$

Substitution of (9) into (4) gives the magnetic critical scattering cross section. The result for a polycrystalline ferromagnet at temperatures above the Curie temperature and for small angles of scattering is

$$
\begin{aligned}
\frac{d \sigma}{d \Omega}=\left(\frac{\gamma e^{2}}{m_{e} c^{2}}\right)^{2} \frac{2 N S(S+1)}{3 \pi r_{1}{ }^{2}} & \frac{k}{k_{0}}|F(K)|^{2} \\
& \times \int \frac{d \omega}{\left(K^{2}+\kappa_{1}^{2}\right)} \frac{\Lambda K^{2}}{\Lambda^{2} K^{4}+\omega^{2}} .
\end{aligned}
$$

There are two approximations in the derivation which restrict (10) to small angles. (a) First, the contributions to the cross section from other points in the reciprocal lattice have been neglected. This is a valid approximation only at angles small enough so that $K \cong 2 \pi \theta / \lambda$ $\ll 2 \pi \tau_{\min }$. (b) The second approximation arises because the first term in the integral (which is the Fourier transform of the ( rnstein-Zernike asymptotic correlation function) only accurately reproduces the transform of the actual spin-correlation function for small $K$.

If the time a neutron spends within the correlation range is small compared to the lifetime of a fluctuation of the spin distribution then $K$ will be essentially independent of $\omega$ and (10) can be easily integrated. The result, in this quasi-static approximation, is a Lorentzian angular distribution

$$
\frac{d \sigma}{d \Omega}=\left(\frac{\gamma e^{2}}{m_{e} c^{2}}\right)^{2} \frac{2 N S(S+1)}{3}|F(K)|^{2} \frac{1}{r_{1}{ }^{2}\left(K^{2}+\kappa_{1}^{2}\right)} .
$$

At temperatures near the Curie temperature $\chi$ is very large; therefore, under the usual conditions of observation $K^{2} \gg \boldsymbol{\kappa}_{1}{ }^{2}$ and it is apparent from (11) that the cross section will be nearly proportional to $(1 / k \theta)^{2}$. Thus the scattering will be most intense when observed with longwavelength neutrons and at small angles.

Equation (10) applies to scattering very close to magnetic Bragg peaks (including the forward direction in the case of a ferromagnet). At larger scattering angles, as mentioned above, deviations can be expected because the Ornstein-Zernike expression only describes the asymptotic behavior of the spin-correlation function. A natural way to extend the angular range of the cross section is to consider further powers of $K^{2}$ in the transform of $\gamma_{R}{ }^{\prime}(0)$, i.e., to represent $\gamma_{R}{ }^{\prime}(0)$ as a sum of increasingly more rapidly damped exponentials

$$
\gamma_{R}{ }^{\prime}(0)=\nu_{\mathrm{a}} e^{-\kappa_{a} R} / R+\nu_{b} e^{-\kappa_{b} R} / R+\nu_{c} e^{-\kappa_{c} R} / R+\cdots,
$$

with $\kappa_{\mathrm{a}} \leqslant \kappa_{b} \leqslant \kappa_{c} \cdots$. Hart ${ }^{20}$ has shown how an extension of the Ornstein-Zernike formula to include at least the next higher power of $K^{2}$ can be justified on thermodynamic grounds. His formula for the correlation function (in slightly modified form) is

$$
\gamma_{R^{\prime}}(0)=\frac{V_{0} S(S+1)}{4 \pi \nu^{2}}\left[\frac{\exp (-\kappa R)}{R}-\frac{\exp (-\eta R)}{R}\right] .
$$

Here $\kappa$ is expected to show a pronounced variation with temperature while $\eta$ remains relatively temperatureindependent. The relation between the susceptibility and the Hart scattering parameters is slightly more complicated,

$$
\nu^{2} \eta^{2} \kappa^{2} /\left(\eta^{2}-\kappa^{2}\right)=\chi_{1} / \chi,
$$

while in the expression for the cross section (10), the term $\left[r_{1}^{2}\left(K^{2}+\kappa_{1}^{2}\right)\right]^{-1}$ is replaced by a term of the form $\left\{\left[\nu^{2} /\left(\eta^{2}-\kappa^{2}\right)\right]\left[K^{4}+\left(\eta^{2}+\kappa^{2}\right) K^{2}+\kappa^{2} \eta^{2}\right]\right\}^{-1}$.

Recently Fisher ${ }^{21}$ has argued that even at small angles there are theoretical limitations on the use of the Ornstein-Zernike function in the immediate neighborhood of the Curie temperature. He suggests that the asymptotic correlation function should be of the form

$$
\gamma_{R^{\prime}}(0)=\frac{V_{0} S(S+1)}{4 \pi r_{1}{ }^{2}} \frac{\exp \left(-\kappa_{0} R\right)}{R^{1+\epsilon}},
$$

where $\epsilon$ is a small number of the order of 0.1. In this formulation, $r_{1}^{2} \kappa_{0}^{2}$ is expected to vary as

$$
\left(\chi^{1-\epsilon / 2}\right)^{-1}
$$

in the neighborhood of the ordering temperature. Substituting (14) into (4) has the effect of changing the term $\left(K^{2}+\kappa_{1}^{2}\right)^{-1}$ in the expression for the cross section (10) to $\left(K^{2}+\kappa_{0}^{2}\right)^{-1+\epsilon / 2}$.

Finally, we want to consider the question of the rela-

${ }^{20}$ E. W. Hart, J. Chem. Phys. 34, 1471 (1961).

${ }^{21}$ M. E. Fisher, J. Math. Phys. 5, 944 (1964). 


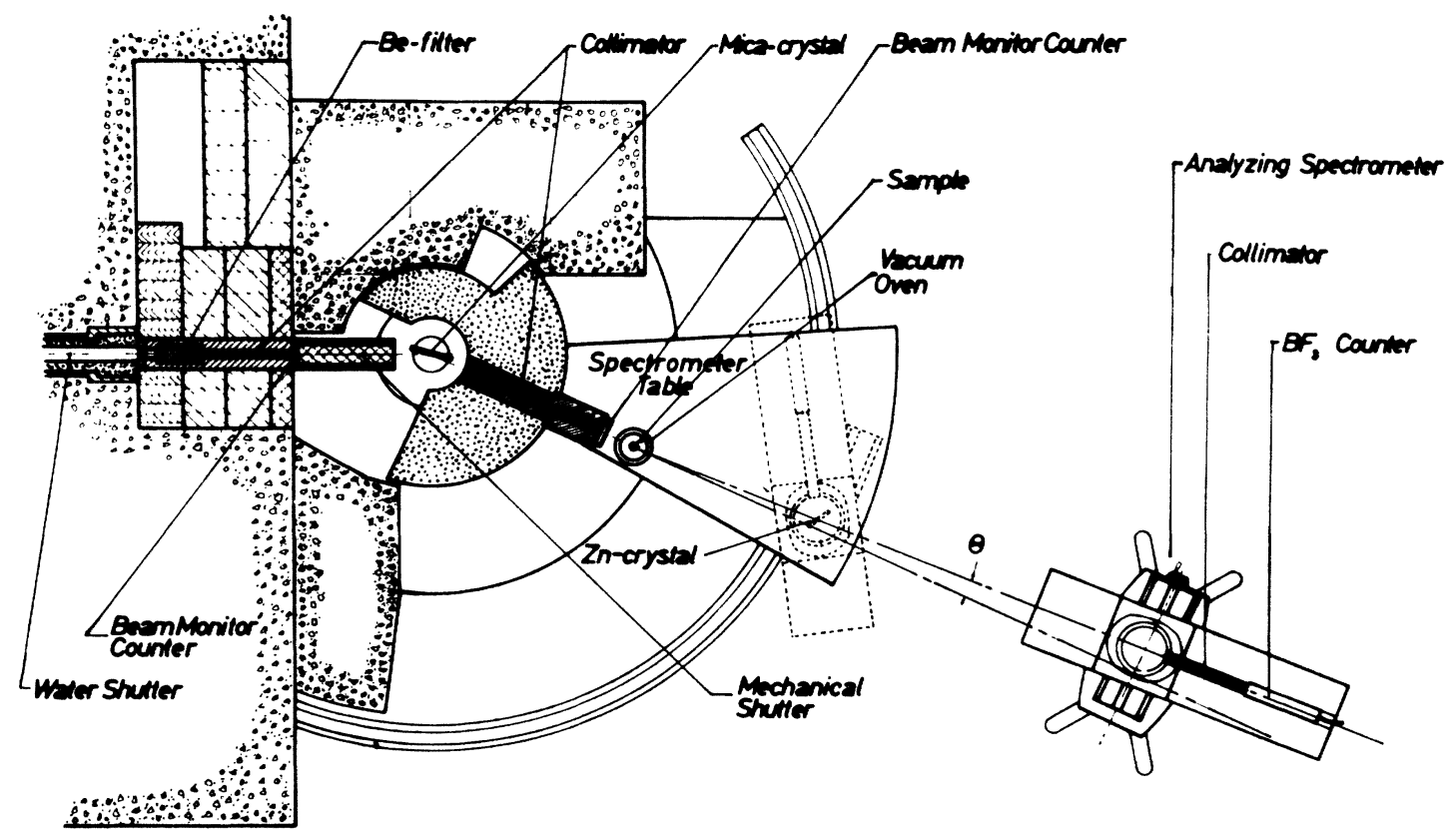

Frg. 1. Schematic showing arrangement of experimental apparatus. The dotted outline shows the spectrometer position when analyzing the energy distribution of the scattered neutrons.

tionship between the exchange parameters describing the microscopic interactions between magnetic ions and the macroscopic neutron-scattering parameters which describe the observed angular and energy distributions. DeGennes ${ }^{18}$ has shown how these relations can be established. The inverse of the static susceptibility is first expressed as a Fourier series

$$
\chi_{1} / \chi=\sum_{\mathbf{R}} \alpha_{\mathbf{R}} \exp (i \mathbf{K} \cdot \mathbf{R}),
$$

where the coefficients $\alpha_{\mathbf{R}}$, which depend only on $|\mathbf{R}|$, are related to the exchange integrals $J(R)$. (At temperatures above $T_{c}$ the coefficients are scalers.) Then, as long as $|\mathbf{K}|$ is small, the exponential term can be expanded to give a series in $\mathbf{K}$.

$$
\begin{aligned}
\chi_{1} / \chi=\sum_{R} \alpha_{R}-\left[(3 !)^{-1}\right. & \left.\sum_{R} R^{2} \alpha_{R}\right] K^{2} \\
& +\left[(5 !)^{-1} \sum_{R} R^{4} \alpha_{R}\right] K^{4}-\cdots .
\end{aligned}
$$

The coefficients of the terms in this series are then directly identified with the neutron-scattering parameters through Eq. (5) above. For example, the parameters of the Ornstein-Zernike function are related to the $\alpha_{R}$ 's by the equations

$$
\begin{aligned}
r_{1}^{2} & =-(3 !)^{-1} \sum_{R} R^{2} \alpha_{R}, \\
\kappa_{1}^{2} r_{1}^{2} & =\chi_{1} / \chi(0)=\sum_{R} \alpha_{R}, \\
\Lambda & =D \chi_{1} / \chi(0)=D \sum \alpha_{R},
\end{aligned}
$$

where $D$ is the intrinsic diffusion coefficient. Equivalent relations will exist for other forms of the correlation function.

It remains to express the $\alpha_{R}$ 's in terms of the exchange integrals. This is in general a difficult problem. The calculations can be done rather easily in the molecular field approximation, however, and give

and

$$
\alpha_{0}=1
$$

$$
\alpha_{R}=-\frac{2}{3}\left(J(R) / K_{B} T\right) S(S+1) .
$$

Thus in this relatively crude approximation the range of the $\alpha_{R}$ 's is indentical to that of the $J(R)$ 's. It is evident that the appearance of terms beyond the order $K^{2}$ in the angular distributions is related to the existence of exchange couplings between more distant neighbors. The molecular-field approximation undoubtedly oversimplifies what is actually a very complex situation and expressions (20) and (21) can only be expected to be correct in a qualitative sense. Other, more realistic methods of doing such calculations have been developed, ${ }^{22-26}$ but discussion of the details of these calculations is beyond the scope of this paper.

\section{DESCRIPTION OF THE EXPERIMENT}

\section{A. Apparatus}

Measurements were made with a modified triple-axis crystal spectrometer at the DR-3 reactor which is of the heavy-water-moderated and -reflected PLUTO type.

${ }^{22}$ R. J. Elliott and W. C. Marshall, Rev. Mod. Phys. 30, 75 (1958)

${ }_{23} \mathrm{H}$. Mori and K. Kawasaki, Progr. Theoret. Phys. (Kyoto) 27, 529 (1962).

${ }^{24}$ T. Izuyama, D. Kim, and R. Kubo, J. Phys. Soc. Japan 18, 1025 (1963).

${ }^{25}$ R. A. Tahir-Kheli and H. B. Callen, J. Appl. Phys. 35, 946 (1964).

${ }^{26} \mathrm{~J}$. Kocinski, J. Phys. Chem. Solids (to be published). 
The arrangement of the apparatus is shown in Fig. 1. The primary beam comes from a light water scatterer located in the reflector in a $6 \times 10^{13} \mathrm{n} / \mathrm{cm}^{2} \mathrm{sec}$ flux. This beam first passed through a $15-\mathrm{cm}$-thick beryllium filter and was then reflected from a mica crystal to produce an intense, monochromatic beam of $4.28 \mathrm{~A}$ neutrons. This wavelength was chosen because it is beyond the crystalline cutoff in iron; hence all Bragg scattering is eliminated. The monochromatic beam, after transmis. sion through a multichannel collimater with 25-min angular divergence, was directed on a sample of highpurity, polycrystalline iron mounted in a vacuum oven. Neutrons scattered by the sample were detected by a shielded $\mathrm{BF}_{3}$ counter with an aluminum oxide end window located on the analyzing spectrometer arm $2.8 \mathrm{~m}$ from the sample. This spectrometer was mounted on rails to permit convenient variation of the angle of observation.

Energy-spectrum measurements were made initially with a crystal analyzing spectrometer using a zinc single crystal in reflection. Later a slow chopper and time-offlight system were also used.

All of the monochromatic beam collimators had titanium plates to eliminate internal reflections.

We investigated the possibility of multiple neutron scattering in the sample distorting the angular distributions by using initially a rod $20 \mathrm{~mm}$ in diameter and $6 \mathrm{~cm}$ high as the sample and then repeating the measurements with the sample reduced to a slab of 12 and finally $4.3 \mathrm{~mm}$ thickness. The results were not appreciably different.

One of the major sources of systematic error in measurements of this type is the sample temperature, which must be extremely uniform and stable. We placed the sample in a water cooled, aluminum jacketed vacuum oven with a heating coil divided into three sections to permit careful adjustment. Surrounding the coil were two cylindrical heat shields made of molybdenum foil. The oven temperature was monitored by a chromelalumel thermocouple connected to a proportional controller which held the temperature constant to $\pm 0.1^{\circ} \mathrm{C}$. Sample temperatures were measured with three calibrated $\mathrm{Pt}-(\mathrm{Pt}, \mathrm{Rh})$ thermocouples placed at the top, middle, and bottom of the sample. To make the temperature distribution as uniform as possible we adjusted the current through the separate sections of the heating coil until the maximum scattered intensity from the top, middle, and bottom regions of the sample coincided. We estimate the temperature gradient to have been no more than $\pm 0.1^{\circ} \mathrm{C}$ at the Curie temperature.

\section{B. Measurements}

A typical set of scattered-neutron angular-distribution measurements made above the Curie temperature is shown in Fig. 2. The scattered intensity was measured at $15 \mathrm{~min}$ angular increments through a range from $1^{\circ}$ to $3 \frac{1}{2}^{\circ}$. At the Curie temperature we extended the measurements out to $9^{\circ}$. When the temperature was

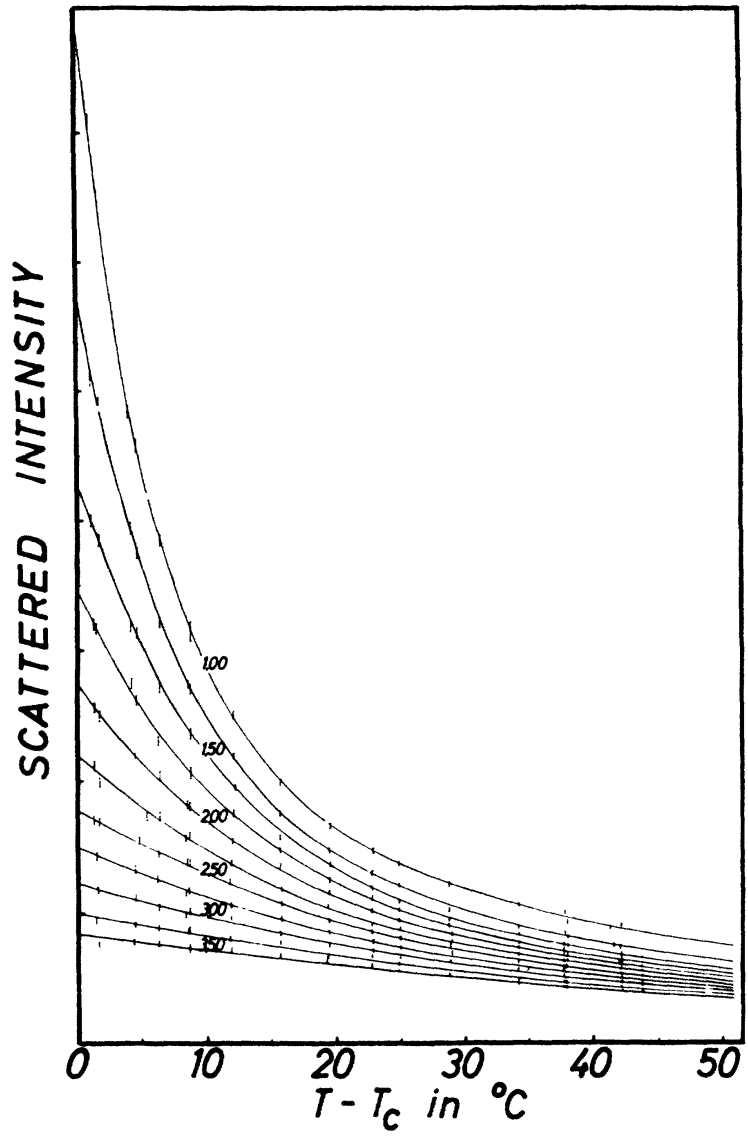

FIG. 2. Variation of scattered intensity with temperature. The numerals give the scattering angle in degrees.

well above the Curie temperature we used a beam which was approximately $6 \mathrm{~cm}$ wide at the sample, however, for measurements very near the Curie temperature, we narrowed the beam to $1 \mathrm{~cm}$ to reduce the effects of any temperature gradients in the sample. There was very little difference in the narrow- and broad-beam data in the overlapping temperature region indicating that the temperature gradient in the sample was small.

We also measured the energy distribution of the incident and scattered neutrons at 2 and $18^{\circ} \mathrm{C}$ above the Curie temperature at scattering angles between 2 and 4 degrees. A typical result appears in Fig. 3 which shows both the incident spectrum and the spectrum at a scattering angle of 2.5 degrees when the sample was $2^{\circ} \mathrm{C}$ above the Curie temperature.

Originally the crystal-analyzing spectrometer was used to measure the spectrum of the beam incident on the sample which is of particular importance to the analysis of the results. However, this led to complications because the wavelength distribution in the beam from the monochromating crystal varies with angle across the beam. ${ }^{27}$

${ }^{27}$ A. H. Compton and S. K. Allison, X-Rays in Theory and Experiment (D. Van Nostrand, New York, 1935), 2nd ed., p. 709. 
Fig. 3. Comparison of incident (solid line) and scattered (dashed line) spectra at $T-T_{c}=2^{\circ} \mathrm{C}$ at a scattering angle of $2.5^{\circ}$.

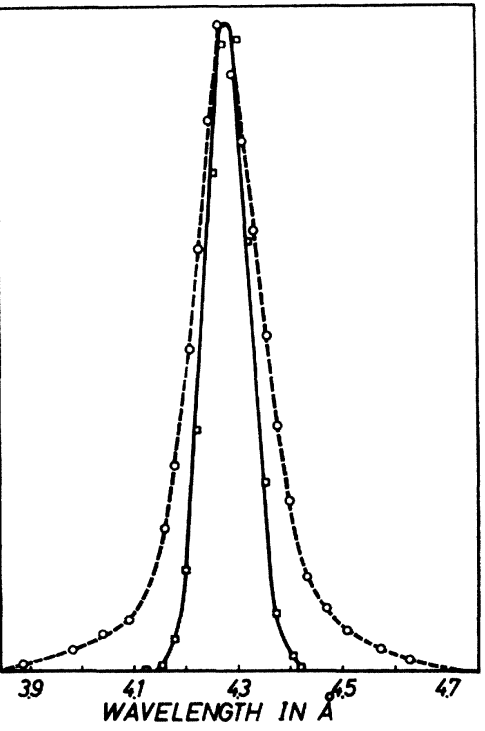

We therefore repeated the measurement with a slow chopper which gives effectively the distribution integrated over all angles in the beam. It is this spectrum, shown in Fig. 3, which we used in the subsequent analysis. Since the scattered neutron distributions do not have so pronounced a variation of wavelength with angle, we measured the scattered spectra with the crystal spectrometer, which had better resolution and gave higher intensity.

\section{ANALYSIS OF THE DATA}

\section{A. Determination of the Scattering Parameters Using the Ornstein-Zernike Correlation Function}

Figure 3 shows clearly that the scattering is not completely elastic. Using the static approximation in analyzing the data is therefore bound to introduce some systematic error. Nevertheless, it is convenient to have preliminary values of the range parameter $\kappa_{1}$ for the subsequent analysis. In Fig. 4 we have plotted the reciprocal of the observed intensity against the square of the scattering angle. It is easy to see from (11) that extrapolating the linear part of these curves to the $\theta^{2}$ axis gives approximate values of $\kappa_{1}$. These approximate values were then used to calculate corrections to the observed angular distributions to take account of both the finite divergence of the incident beam and the angular resolution of the analyzing collimation system. The angular corrections were mostly associated with the 40 min vertical divergence of the incident beam necessary for reasons of intensity at the higher temperatures. These corrections, while small at temperatures well above $T_{c}$, became of increasing importance at temperatures near $T_{c}$.

To determine more accurate values of $\kappa_{1}$ we fitted curves calculated from the static approximation form of the cross section to the corrected, observed distri-
TABLE I. Values of the spin-correlation range $\kappa_{1}^{-1}$ obtained using the Van Hove theory in the static approximation, and with the time dependence included. The third column shows the effect on $\kappa_{1}^{-1}$ of the modification suggested by Fisher.

\begin{tabular}{|c|c|c|c|}
\hline \multirow[b]{3}{*}{$T-T$ in ${ }^{\circ} \mathrm{C}$} & \multicolumn{3}{|c|}{ Spin-correlation range in $\AA$} \\
\hline & \multicolumn{2}{|c|}{ Ornstein-Zernike } & \multirow{2}{*}{$\begin{array}{c}\text { Fisher } \\
2 m \Lambda / \hbar=11 \\
\epsilon=0.15\end{array}$} \\
\hline & $\begin{array}{c}\text { Static } \\
\text { approximation }\end{array}$ & $2 m \Lambda / \hbar=11$ & \\
\hline 1.5 & 55.2 & 40.7 & 55.6 \\
\hline 2.0 & 45.9 & 35.5 & 44.9 \\
\hline 3.5 & 37.9 & 30.3 & 36.4 \\
\hline 5.0 & 32.7 & 26.7 & 30.8 \\
\hline 7.5 & 26.7 & 22.0 & 24.7 \\
\hline 10.0 & 23.0 & 19.1 & 21.0 \\
\hline 12.5 & 20.1 & 16.5 & 18.0 \\
\hline 15.0 & 18.1 & 14.8 & 16.0 \\
\hline 17.5 & 16.6 & 13.4 & 14.5 \\
\hline 20.0 & 15.2 & 12.1 & 13.0 \\
\hline 30.0 & 12.1 & 9.1 & 9.7 \\
\hline 40.0 & 10.5 & 7.6 & 8.0 \\
\hline 50.0 & 10.0 & 7.0 & 7.4 \\
\hline 60.0 & 9.0 & 6.0 & 6.3 \\
\hline
\end{tabular}

butions. These values are listed in the first column of Table $I$ and are plotted in Fig. 7.

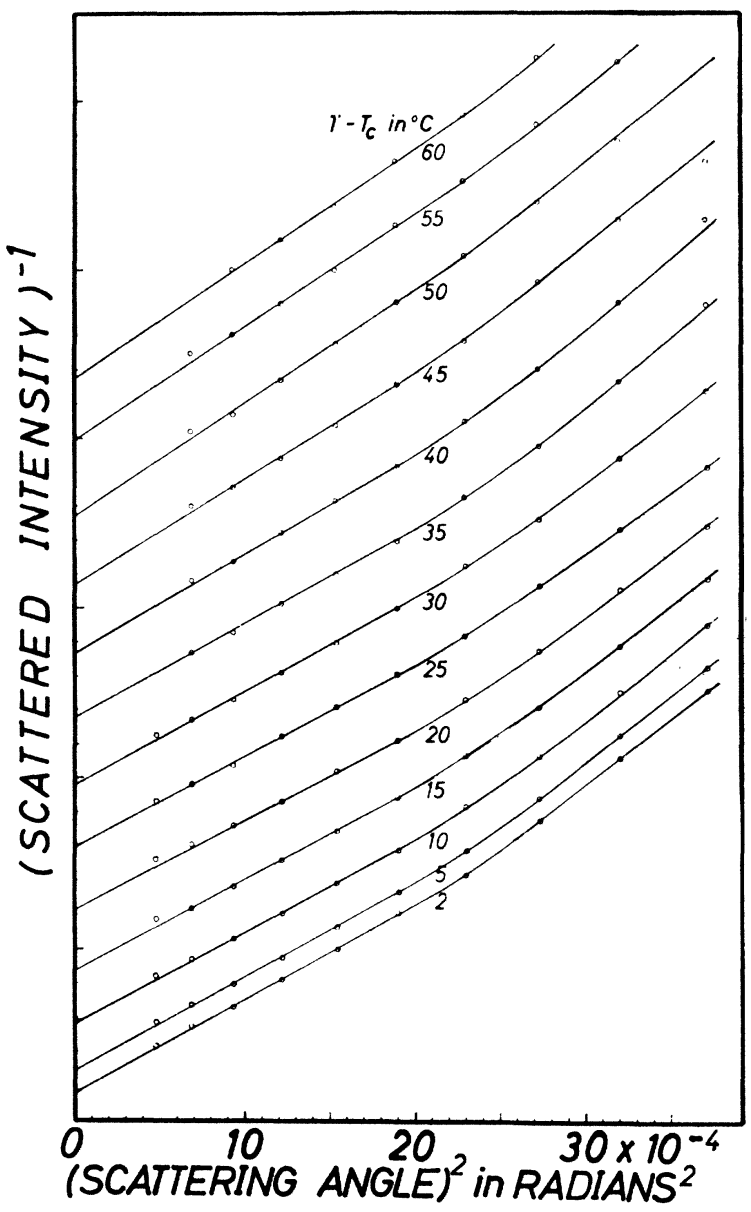

FIG. 4. Reciprocal of the observed scattered intensity plotted against square of scattering angle to show the Lorentzian-like form of the angular distributions. 


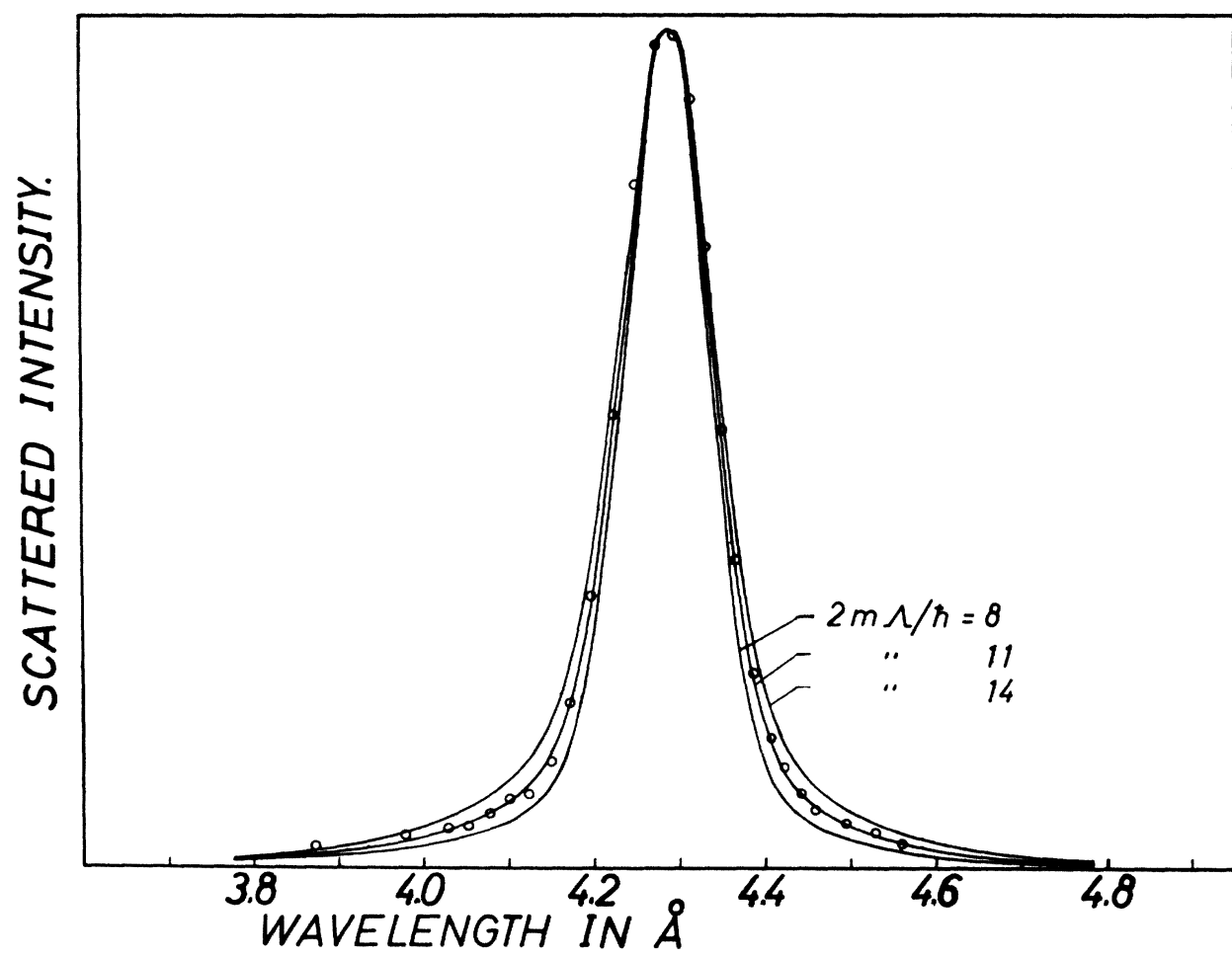

Fig. 5. Comparison of the observed scattered spectrum at $\theta=2^{\circ}$ and $T-T_{c}=18^{\circ} \mathrm{C}$ with curves calculated for various values $2 m \Lambda / \hbar$.
Fitting the data to the more exact expression (10) for the cross section was done in two steps. First, using the static approximation values for $\kappa_{1}$, we calculated the spectral distributions to be expected for various values of $\Lambda$ by folding the measured incident spectrum with the cross section, (10). A typical set of these calculated distributions is shown in Fig. 5. To find the best value of $\Lambda$, the calculated width of the spectrum at halfmaximum was compared with the measured value as shown in Fig. 6.

Within the limits of error, the same value of the dimensionless parameter $2 m \Lambda / \hbar=11.0 \pm 0.6$ fitted both the data at $T-T_{c}=2^{\circ} \mathrm{C}$ and $T-T_{c}=18^{\circ} \mathrm{C}$. The quoted error is estimated from the fit of the data to the calculated width and does not include any estimate of possible systematic errors in the spectrum measurements. Varying $\kappa_{1}$ had almost no effect on the spectrum calculations, hence using the static approximation values for $\kappa_{1}$ did not make any significant difference to the results.

Although these calculations had to be done numerically with a computer, we were able to check some of the results analytically as well. At small angles the scattered distribution for monochromatic incident neutrons is very nearly a Lorentzian with a full width at halfmaximum of $2 m \Lambda \theta^{2} / h$. Our incident spectrum was close to being Gaussian in shape, hence the tabulated Voigt integral, ${ }^{28}$ which folds a Gaussian with a Lorentzian, should give a close approximation to our numerical

${ }^{28}$ J. T. Davies and J. M. Vaughan, Astrophys. J. 137, 1302 (1963). calculations. The agreement at small angles was excellent. As the scattering angle increases, however, the approximate expression $2 m \Lambda \theta^{2} / \hbar$ increasingly overestimates the actual width of the scattered distribution. Therefore, applying the Lorentzian approximation at larger angles leads to an underestimate of the value of $\Lambda$.

After determining $\Lambda$ we calculated the angular distributions predicted by (10) and found the values of $\kappa_{1}$ and $r_{1}^{2}(T) / r_{1}^{2}\left(T_{c}\right)$ which gave the best weighted leastsquares fits to the observed distributions. We used only

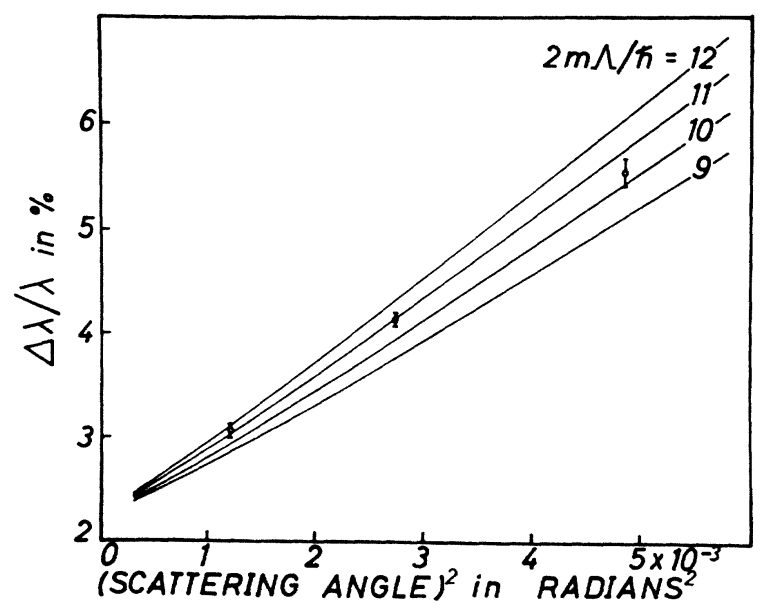

FIG. 6. Comparison of the observed full width of the scattered spectrum at half-maximum at $T-T_{\mathrm{c}}=18^{\circ} \mathrm{C}$ with curves calculated for various values of $2 m \Lambda / \hbar$. The calculations were made with the $\kappa_{1}^{-1}=12.2 \AA$ 
the data from scattering angles between 1.5 and 2.75 degrees, i.e., the linear region in Fig. 4. The values obtained for $\kappa_{1}$ are given in the second column in Table I and are plotted in Fig. 7. The ratio $r_{1}^{2}(T) / r_{1}{ }^{2}\left(T_{c}\right)$ is plotted in Fig. 8.

The fractional standard deviations of the values of $\kappa_{1}$ were of the order of $2 \%$, while for the ratio $r_{1}^{2}(T) / r_{1}{ }^{2}\left(T_{c}\right)$ the fractional standard deviations were about $3 \%$. This represents only statistical uncertainties and does not include possible systematic errors such as the effects of multiple scattering or errors introduced by approxima-

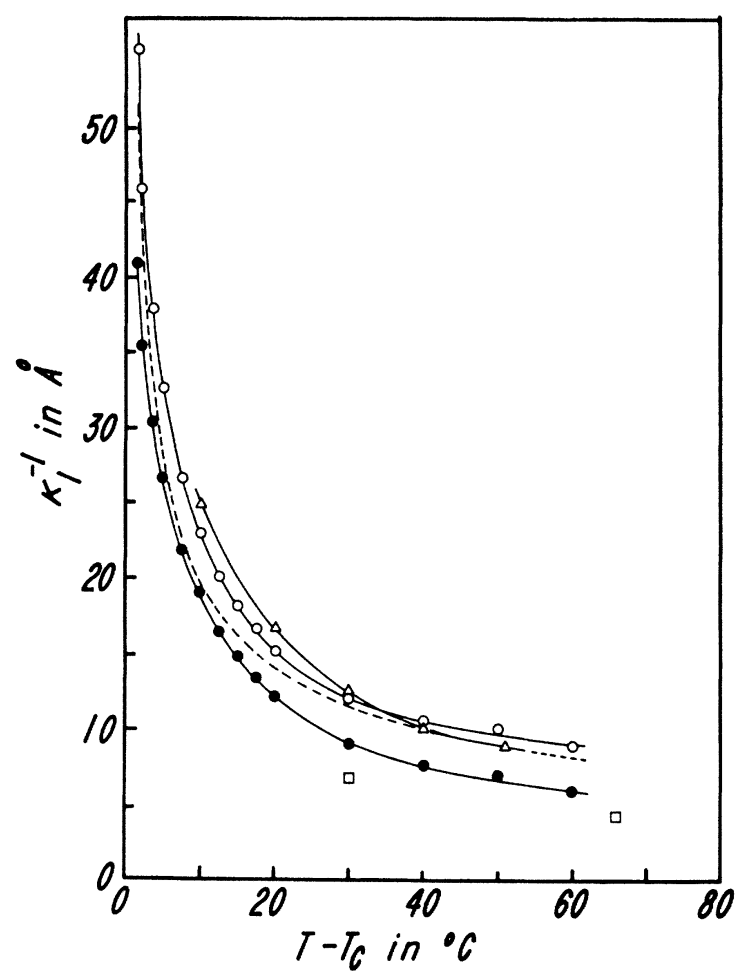

Fig. 7. Variation of spin-correlation range $\kappa_{1}^{-1}$ with temperature. The open circles are the results of the present measurements analyzed in the static approximation, while the solid circles show the effect of including the inelasticity in the data analysis. The triangles and squares are the results of Jacrot et al., (Ref. 13) and Gersch et al., (Ref. 7), respectively, analyzed assuming the static approximation. The dashed line is a recent calculation by Kocinski (Ref. 26).

tions in the angular resolution corrections. Unfortunately, it has proven to be very difficult to estimate the limits of such errors; however, we believe they are of the same order of magnitude as the statistical errors.

It is apparent that the values for $\kappa_{1}^{-1}$ resulting from fitting the data to (10) are significantly smaller than the values obtained from the static approximation. This is due to the fact that in the static approximation the integration over $\omega$ gives simply $\pi /\left(K^{2}+\kappa_{1}^{2}\right)$. In fact, the values of the integral with $2 m \Lambda / h=11$ is somewhat larger than this at small angles and decreases as the angle increases. This has the effect of changing the

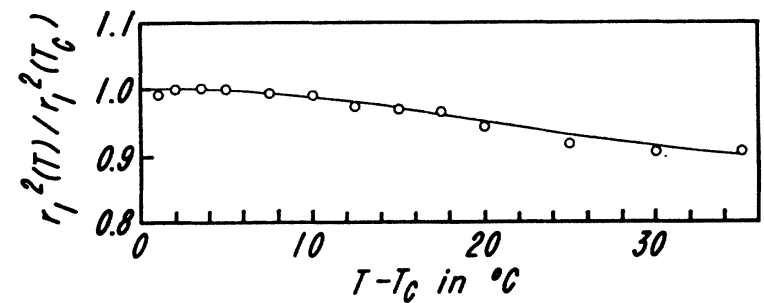

FIg. 8. Variation of $r_{1}^{2}(T) / r_{1}{ }^{2}\left(T_{c}\right)$ with temperature.

slope and therefore the intercept of the static-approximation curves.

\section{B. Determination of the Scattering Parameters Using Hart's Correlation Function}

The procedure followed was essentially the same as described in IV.A. We calculated the angular distributions using the appropriate form of the cross section with $2 m \Lambda / h=11$ and found the values of $\kappa$ and $\eta$ which gave the best least-squares fit to the extended measurements made at the Curie temperature. In Fig. 9 we show the resulting fit to the observed angular distribution obtained with $\kappa=0.0181 \AA^{-1}$ and $\eta=0.184 \AA^{-1}$.

\section{Determination of the Scattering Parameters Using Fisher's Correlation Function}

Again the procedure followed was the same as in IV.A. The angular distributions were calculated

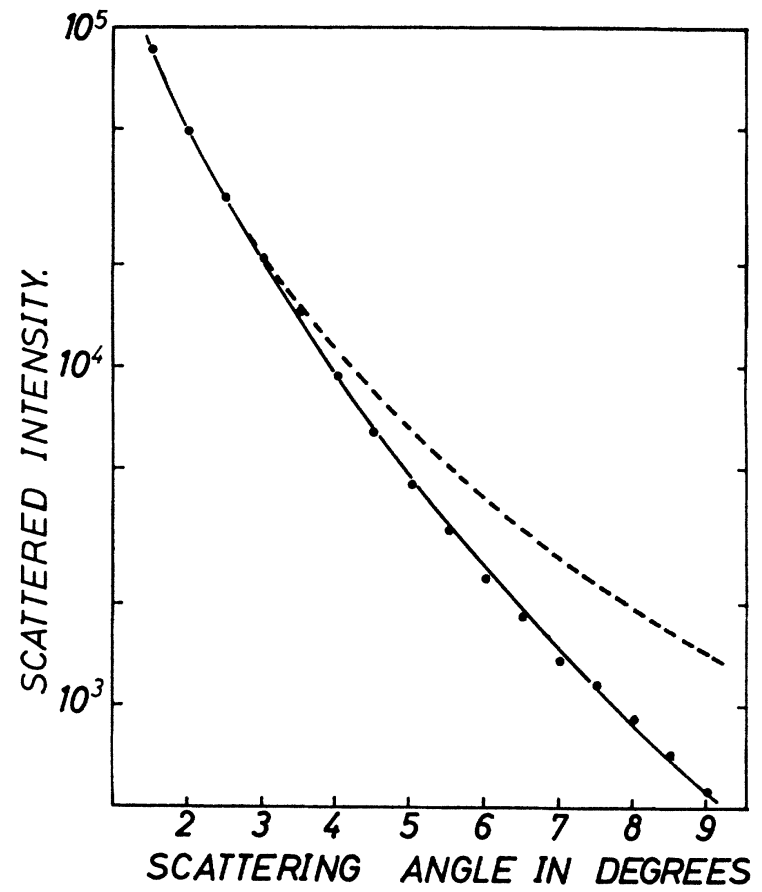

FIG. 9. Comparison of scattered intensity slightly above the Curie temperature with calculated distributions. The solid line was calculated using the Hart correlation function with $\kappa=0.0181$ $\AA^{-1}$ and $\eta=0.184 \AA^{-1}$. The dashed line was calculated using the Ornstein-Zernike correlation function with $\kappa_{1}=0.00583 \AA^{-1}$. In both calculations $2 m \Lambda / \hbar=11$. 
from (10) but with the term $\left(K^{2}+\kappa_{1}^{2}\right)^{-1}$ replaced by $\left(K^{2}+\kappa_{0}^{2}\right)^{-1+(1 / 2) \epsilon}$ We used $\epsilon=0.10,0.15$, and 0.20 and, as before, $2 m \Lambda / \hbar=11$. The calculated curves fitted the observed distributions about as well as with the Ornstein-Zernike function, ${ }^{29}$ however, for $\epsilon>0.15$ the strength parameter $r_{1}$ showed a tendency to increase with increasing temperature contrary to what would be expected on physical grounds. The results for $\kappa_{0}$ obtained with $\epsilon=0.15$ are given in the last column of Table I.

\section{DISCUSSION OF THE RESULTS}

\section{A. The Temperature Dependence of the Spin-Correlation Range}

Previous to the recent experiments of Jacrot and his collaborators, measurements of critical neutron scattering were analyzed in terms of the static approximation. It would be misleading to compare our results as presently analyzed with earlier measurements, but it is still instructive to see to what extent the angular distributions we observed agree with those obtained by other groups. We therefore compare in Fig. 7 the values we obtained for $\kappa_{1}$ in the static approximation with those obtained by Jacrot et al., ${ }^{13}$ and Gersch, Shull, and Wilkinson. ${ }^{7}$ Obviously, there is considerable disagreement on the magnitude of $\kappa_{1}$ as determined by different groups although our values appear to be in reasonable agreement with those obtained by Jacrot and his collaborators.

Since the neutron-scattering parameters are directly related to the zero-field magnetic susceptibility, it is of interest to see to what extent these measurements confirm Domb and Sykes ${ }^{14}$ and Gammel, Marshall, and Morgan' $\mathrm{s}^{15}$ prediction that the susceptibility varies as $\left(T-T_{c}\right)^{-\alpha}$ where $\alpha=\frac{4}{3}$ in the neighborhood of the Curie temperature. For $T-T_{c}>\sim 5^{\circ} \mathrm{C}$ we find that $T\left(\kappa_{1} r_{1}\right)^{2}$, which is inversely proportional to the susceptibility, is an excellent fit to a function of the form $\left(T-T_{c}\right)^{\alpha}$ with $\alpha=1.30 \pm 0.04$ as can be seen in Fig. 10. Using the modified theory of Fisher does not change the exponent significantly. Regardless of whether $\epsilon=0.10,0.15$, or 0.20 we find $\alpha$ has essentially the same value, $1.29 \pm 0.04$.

At temperatures close to $T_{c}$ Fig. 10 indicates a departure from the $\frac{4}{3}$ law. Fisher has predicted that this would be expected as a consequence of the limitations of the Ornstein-Zernike theory. In Fisher's formulation it is the modified function $\left(\kappa_{0} r_{1}\right)^{2-\epsilon}$ which is related to $1 / \chi$. Indeed, using his form of the correlation function does improve the agreement in the neighborhood of $T_{c}$. We are not certain, however, that this should really be interpreted as evidence in support of Fisher's ideas. There are a number of experimental factors, all of them

20 This in contradiction with our earlier conclusion reported in J. Appl. Phys. Suppl. 35, 933 (1964). At that time we based our analysis on angular distributions which were not corrected for angular divergence. We are indebted to $\mathrm{Dr}$. $\mathrm{T}$. Riste for pointing out that the effect of angular divergence was larger than we had anticipated.

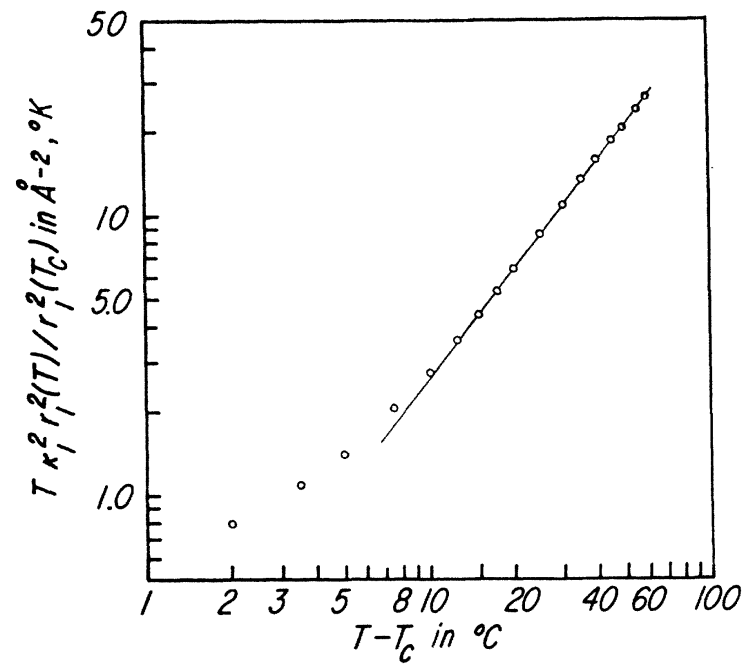

FIG. 10. Plot of logarithm of $T\left(\kappa_{1} r_{1}\right)^{2}$ against the logarithm of $T-T_{c}$. The slope of the line is 1.30 .

difficult to assess, which could be equally responsible. First, as was mentioned above, the effects of angular divergence become increasingly important at temperatures near $T_{c}$. In this temperature region the data analysis is very sensitive to the angular resolution and to the finite energy spread in the incident beam. It is not unreasonable to suspect that at least part of the deviation from the $\frac{4}{3}$ law could be simply a consequence of approximations used in making these corrections. Also a small temperature gradient or multiple scattering in the sample could distort the distributions near the Curie temperature. Therefore, we do not feel that these data should be put forward as a definitive test of Fisher's ideas although they are certainly not inconsistent with his conclusions.

If it is assumed that $\alpha=\frac{4}{3}$, extrapolation of the higher temperature data indicates a nonzero value of the range parameter $\kappa_{1}$ and a finite value of the susceptibility at the Curie temperature (see Ref. 29). This is contrary to expectations and is in fact simply the result of a logical inconsistency since our measurements indicate $\alpha=1.30$. Extrapolation with this value of the exponent leads to an infinite susceptibility as expected. Thus if there is any experimental evidence of a finite value of the susceptibility at the Curie temperature it is contained in the data close to $T_{c}$ where, as we have seen, the interpretation becomes very difficult.

\section{B. Comparison of the Scattering Parameters with Susceptibility Measurements}

Direct comparison of our results with the existing magnetic-susceptibility data is useful as a test of the internal consistency of these two very different methods of measurement. As far as the temperature dependence is concerned, the recent susceptibility measurements of Noakes and Arrott ${ }^{16}$ at $T \gtrsim T_{c}$ give $\alpha=1.37 \pm 0.04$. Arajs 
and Colvin ${ }^{17}$ also found $\alpha=\frac{4}{3}$ from $T_{c}$ to $T_{c}+30$ in good agreement with our results. Unfortunately, other susceptibility measurements at higher temperatures ${ }^{30-32}$ do not provide a very precise indication of the temperature variation so no comparison is possible.

In order to intercompare the magnitudes of the scattering parameters with the susceptibility, it is necessary to know both $r_{1}$ and $\kappa_{1}$ absolutely. While the absolute value of $\kappa_{1}$ can be obtained directly from the angular distributions, this is not the case with $r_{1}$ which can only be determined relatively unless the magnitude of the cross section is also measured. Several such cross-section measurements have been made, however. Gersch et al. ${ }^{7}$ obtained for $r_{1}$ at the Curie temperature $1.05 \pm 0.05 \AA$, while Ericson and Jacrot ${ }^{11}$ found $1.46 \AA$. The reasons for this large difference do not appear to have been resolved. In any case we will take the median value, $1.26 \AA$, and use Eq. (7) to make an intercomparison at for example $T-T_{c}=30^{\circ}$. Using Fig. 8 to correct for the temperature dependence of $r_{1}$, we obtain $r_{1}^{2} \kappa_{1}{ }^{2}=0.018$ with an uncertainty of about $20 \%$.

There is a considerable spread in the values of the susceptibility as reported by various investigators. A rough average of the values of $\chi_{1} / \chi$, measured at $T-T_{c}$ $=30^{\circ}$, is 0.015 with an uncertainty of about the same magnitude. ${ }^{7}$ This establishes that at least on an orderof-magnitude basis the neutron measurements are consistent with previous magnetic measurements.

\section{Corrections to the Lorentzian Angular Distribution}

Inspection of Figs. 4 and 9 shows that the observed angular distributions depart noticeably from the Lorentzian shape at larger scattering angles. Clearly the fit of the calculated distributions to the data is greatly improved by including a $K^{4}$ term in the differential cross section. As we have seen, this is an indication of the importance of couplings of longer range in the ordering of the spin system. The existence of long-range couplings in a metal is not unexpected and in fact a $K^{4}$ term has also been observed in the scattering from nickel by Cribier, Jacrot, and Parette..$^{33}$ However, $1 /\left(\eta^{2}+\kappa^{2}\right)$, which is the ratio of the coefficients of the $K^{4}$ and $K^{2}$ terms in the angular distribution, is $29 \AA^{2}$; considerably smaller than the value of $110 \AA^{2}$ reported for nickel. As far as we could determine this ratio remains constant from $T-T_{c}=0$ to $10^{\circ} \mathrm{C}$.

\section{Dynamics of the Fluctuations}

There is clear evidence that the scattering is appreciably inelastic both in the immediate neighborhood of

${ }^{30} \mathrm{~W}$. Sucksmith and R. R. Pearce, Proc. Roy. Soc. (London) A167, 189 (1938).

${ }^{21}$ S. Arajs and S. D. Miller, J. Appl. Phys. 31, 986 (1960).

32 H. H. Potter, Proc. Roy. Soc. (London) A146, 362 (1934)

${ }^{33}$ D. Cribier, B. Jacrot, and G. Parette, J. Phys. Soc. Japan 17,67 (1962). The value quoted in this reference is $200 \AA^{2}$. A later re-analysis of the data [B. Jacrot (unpublished)] gave $110 \AA^{2}$. the Curie temperature and at higher temperatures. In fact our measurements show that $2 m \Lambda / \hbar$ has essentially the same value, $11.0 \pm 0.6$, at $T-T_{c}=2$ and $18^{\circ} \mathrm{C}$. These results are in excellent agreement with those obtained by Jacrot and his collaborators ${ }^{13}$ who found $2 m \Lambda / \hbar$ $=11.4 \pm 1.0^{34}$ without any significant variation up to $T-T_{c}=30^{\circ} \mathrm{C}$.

While the experimental results obtained by different groups appear to be consistent among themselves, they are not at all consistent with theoretical expectations. For if $\Lambda$ is inversely proportional to the susceptibility as Van Hove assumed, it should be zero at the Curie point and should vary approximately as $1 / \chi$ in the neighborhood of $T_{c}$. Mori and Kawasaki ${ }^{35}$ and DeGennes and Villain ${ }^{36}$ have re-examined this question but their conclusions are not substantially different from Van Hove's. Recently, however, Kocinski ${ }^{37}$ has found that by applying irreversible thermodynamics to the description of the dynamics of the fluctuations he can predict the observed temperature dependence of $\Lambda$ quite well and even calculate its magnitude with a fair degree of accuracy.

\section{SUMMARY}

The study of slow neutron scattering has given us considerable insight into the details of the magnetic ordering process in iron. We have demonstrated that the expected relationship between the static magnetic susceptibility and the parameters which describe the neutron scattering does in fact exist. And, by studying the temperature variation of the neturon-scattering parameters, we have been able to show that the zerofield susceptibility varies as $\left(T-T_{c}\right)^{-1.30 \pm 0.04}$ up to a temperature approximately $60^{\circ} \mathrm{C}$ above the Curie temperature. This is in substantial agreement with recent predictions for a Heisenberg ferromagnet.

There is evidence, unfortunately, of an integral rather than differential nature, that magnetic interactions in iron extend beyond the nearest neighbors. However, longer range interactions appear to be considerably less important in iron than in nickel. This is possibly why a theoretical model which includes only exchange between nearest neighbors gives results in such good agreement with experiment.

Unexpectedly, the time constant associated with the decay of the spin fluctuations is found to be independent of the temperature in the neighborhood of the Curie temperature. Whether this can be reconciled with the existing theories does not seem clear at the moment.

Our attempts to look for certain small departures

24 The value originally quoted in Ref. 13 was $2 m \Lambda / \hbar=7$, based on an approximate treatment of the data. We have recently been advised by G. Parette that a more exact numerical analysis of their data gives the value $2 m \Lambda / \hbar=11.4$.

${ }^{35} \mathrm{H}$. Mori and K. Kawasaki, Progr. Theoret. Phys. (Kyoto) 27, 529 (1962).

${ }^{36}$ P. D. DeGennes and J. Villain, J. Phys. Chem. Solids 13, $10(1960)$.

${ }_{27}$ J. Kocinski, Acta Phys. Polon. 24, 273 (1963). 
from the Ornstein-Zernike theory in the neighborhood of the Curie temperature were of limited success. While we did observe anomalies in the data in this temperature region, we were not able to establish that they were the expected effects. This question certainly warrants further investigation if apparatus of improved resolution becomes feasible in the future.

\section{ACKNOWLEDGMENTS}

We wish to express our appreciation for the continued support and encouragement of Professor C tto KofoedHansen and Professor H. Højgaard-Jensen. It is also a pleasure to acknowledge our obligation to Dr. Walter
Marshall who originally directed our attention to this interesting field. Dr. M. E. Fisher and Dr. E. W. Hart gave very generously of their time to discuss details of their work with one of us (L.P.) and Dr. J. Kocinski was kind enough to communicate some of his calculations to us in advance of publication. We also wish to take this opportunity to thank our colleagues, W. K. Kofoed who was responsible for the design and construction of the apparatus, F. Hansen who designed the oven control system, and D. Heidebo who rendered valuable assistance. The numerical calculations were performed on the GIER-computer of Afdeling for Numerisk Analyse, University of Copenhagen.

\title{
Magnetoacoustic Effect in Rhenium near $1 \mathrm{Gc} / \mathrm{sec}^{*}$
}

\author{
C. K. JoNES \\ Westinghouse Research Laboratories, Pittsburgh, Pennsylvania \\ AND \\ J. A. RAYNe \\ Carnegie Institute of Technology, Pittsburgh, Pennsylvania \\ (Received 19 April 1965)
}

\begin{abstract}
Magnetoacoustic measurements near $1 \mathrm{Gc} / \mathrm{sec}$ have been made in very pure rhenium. The resulting extremal dimensions are compared with the Fermi surface model deduced from low-field de Haas-van Alphen data. Reasonable agreement is found for the dumbbells of the model, but there appears to be some difficulty with respect to the ellipsoids. Possible reasons for this discrepancy are considered. Dimensions corresponding to large pieces of the Fermi surface are also observed and are tentatively correlated with the results of pulsed-field de Haas-van Alphen data.
\end{abstract}

\section{INTRODUCTION}

$T$ HERE is a considerable body of theoretical and experimental work concerning many of the cubic transition metals. Relatively little information, however, is available about the transition elements of hexagonal symmetry. Band calculations exist only for titanium $^{1}$ and zirconium, ${ }^{2}$ and experimental data relating directly to the nature of the Fermi surfaces of hexagonal transition metals is quite sparse. In this respect rhenium is somewhat exceptional in that both high- and low-field de Haas-van Alphen ${ }^{3,4}$ as well as magnetoresistance ${ }^{5}$ data are available, and that crystals of high purity are readily obtainable. In this paper, we report magnetoacoustic measurements up to $930 \mathrm{Mc} / \mathrm{sec}$ on single-crystal rhenium having a resistance ratio in

\footnotetext{
* Supported in part by the National Science Foundation. 1 S. L. Altmann and N. V. Cohen, Proc. Phys. Soc. (London) 71, 383 (1958).

${ }^{2}$ S. L. Altmann and C. J. Bradley, Phys. Letters 1, 336 (1962).

${ }^{3}$ A. C. Thorsen and T. G. Berlincourt, Phys. Rev. Letters 7, 244 (1961).

${ }^{4}$ A. S. Joseph and A. C. Thorsen, Phys. Rev. 133, A1547 (1964)

${ }^{5}$ W. A. Reed, E. Fawcett, and R. R. Soden, Phys. Rev. 139, A1557 (1965).
}

excess of 20000 to 1 . In the absence of any theoretical band structure, a comparison of the extremal dimensions is made with the model deduced from de Haas-van Alphen (dHvA) data.

\section{EXPERIMENTAL TECHNIQUE}

The rhenium crystal used in this work was grown in an electron-beam furnace by $\mathrm{H}$. Sell of the Westinghouse Lamp Division, Bloomfield, N. J. An accurate figure for the resistance ratio of the resulting monocrystal is not available, but it is believed to be in excess of 20000 to 1. Samples suitable for ultrasonic measurements, oriented with faces perpendicular to [0001], [1010], and $[1 \overline{2} 10]$, were spark cut from the crystal. The opposite ends of each sample were subsequently lapped for parallelism to within $0.0001 \mathrm{~cm}$ and flatness to within a fringe over their entire surfaces. The samples were then indium bonded to $z$-cut quartz delay lines, approximately one inch in length.

Magnetoacoustic measurements up to $930 \mathrm{Mc} / \mathrm{sec}$ were made on these composite specimens, using the 